\title{
Improving incident reporting among junior doctors
}

BMJ Quality Improvement Reports 2014; u202381.w2481 doi: 10.1136/bmjquality.u202381.w2481

\author{
Emily Hotton, Lesley Jordan, Carol Peden
}

Royal United Hospital Bath, UK

\begin{abstract}
To ensure systems in hospitals improve to make patient care safer, learning must occur when things go wrong. Incident reporting is one of the commonest mechanisms used to learn from harm events and near misses. Only a relatively small number of incidents that occur are actually reported and different groups of staff have different rates of reporting. Nationally, junior doctors are low reporters of incidents, a finding supported by our local data. We set out to explore the culture and awareness around incident reporting among our junior doctors, and to improve the incident reporting rate within this important staff group. In order to achieve this we undertook a number of work programmes focused on junior doctors, including: assessment of their knowledge, confidence and understanding of incident reporting, education on how and why to report incidents with a focus on reporting on clinical themes during a specific time period, and evaluation of the experience of those doctors who reported incidents.
\end{abstract}

Junior doctors were asked to focus on incident reporting during a one week period. Before and after this focussed week, they were invited to complete a questionnaire exploring their confidence about what an incident was and how to report.

Prior to "Incident Reporting Week", on average only two reports were submitted a month by junior doctors compared with an average of 15 per month following the education and awareness week. This project highlights the fact that using a focussed reporting period and/or specific clinical themes as an education tool can benefit a hospital by promoting awareness of incidents and by increasing incident reporting rates. This can only assist in improving hospital systems, and ultimately increase patient safety.

\section{Problem}

To ensure systems in hospitals improve to make patient care safer, learning must occur when things go wrong.[1] Junior doctors are at the frontline of patient care and are likely to witness errors and system failures. However, doctors as a profession are low reporters of incidents compared with nurses[2,3]; there may be many reasons for this including fear of blame, lack of knowledge of how to report an incident, lack of clarity around what constitutes an "incident" and lack of belief that reporting has value, often related to poor feedback systems. [4,5] We recognised that junior doctors were low reporters of incidents in our hospital (Royal United Hospital Trust, Bath) and set out to explore this and improve the system and reporting culture within our Trust.

\section{Background}

Incident reporting is an established mechanism for improving safety through learning from failures in health care and in industry.[6,7] Incident reporting is widely used and promoted as an effective tool to make an organisation safer.[4,5,6,7] Hospitals that report large numbers of incidents are viewed as having an effective safety culture.[8] However, it is recognised that only a relatively small percentage of incidents that occur are actually reported, and crucially those incidents where harm did not occur but where learning could be significant to prevent future harm ("near misses") are not reported.[5,9] The reasons for failure to report incidents have been extensively analysed and include: time constraints; failure to recognise an incident, fear of blame, failure to receive feedback, and lack of belief in the value of the system.[4,5,9,10,11] On the basis of these issues, the World Health Organization has convened work to improve development and management of patient safety incident reporting systems.[6]

Doctors have low rates of incident reporting compared with other staff groups.[2,3,5] Traditionally medical education has focused on morbidity and mortality reviews to promote learning around how care could be improved. However, these meetings are frequently only attended by doctors and focus on individual performance and not system improvement; the lessons learnt remain within the medical team and are not shared with the whole hospital system where they could promote wider learning and identification of those system issues that might compromise safety.[12,13]

The need for improvement in the culture around healthcare incident reporting is often illustrated by comparison with high reliability organisations such as nuclear power and commercial aviation.[7] Incidents are reliably reported by all team members, analysed often by an independent team, and the results and learning are widely disseminated using a variety of mechanisms. In addition, the "WHO Draft Guidelines for Adverse Event Reporting and Learning Systems" published in 2005 outlines the need for a transferable reporting system that would allow for comparisons of incident reporting between different healthcare systems, a call that has been widely echoed.[14,15]

There has been increasing recognition that junior doctors are important members of the safety team in a hospital. The report by Sir Bruce Keogh (medical director of the NHS) on hospitals with 
high mortality rates stated eight ambitions for improvement, one of which was "Junior doctors in specialist training will not just be seen as the clinical leaders of tomorrow, but clinical leaders of today. The NHS will join the best organisations in the world by harnessing the energy and creativity of its 50,000 young doctors."[16]

\section{Baseline measurement}

Incident reporting rates for junior doctors (in this project $\mathrm{F} 1$ and $\mathrm{F} 2$ doctors) were extracted and analysed on a monthly basis from the Datix incident reporting system for the seven months prior to the improvement period.

\section{Design}

In order to improve the culture around incident reporting in our organisation we undertook a number of work programmes to improve incident reporting rates and the feedback from incident reporting. We sought to specifically increase junior doctors' exposure to incident reporting in a number of ways:

- Assessing junior doctors' knowledge, confidence, and understanding of incident reporting

- Educating junior doctors about how and why to report

- Selecting clinical areas and themes that were important to junior doctors and focusing on reporting on these

- Encouraging junior doctors to actively report incidents in the selected areas or safety themes during a specific time period of one week

- Evaluating the experience of junior doctors who reported incidents

These points were decided on following discussion with junior doctors, senior clinicians, and the hospitals Datix team. We wanted to ensure that the junior doctors were given focussed and comprehensive exposure on how to report an incident, to help them understand why it is important, how it is beneficial to patients, and how it can be used to improve hospital systems. In this study we chose not to focus on the feedback of incidents; we are well aware of the importance of this aspect of the incident reporting loop, although we decided that would be the subject of a follow up project.

\section{Strategy}

During a one week period (2nd March 2014 to 9th March 2014), junior doctors were asked to focus on incident reporting. Prior to this week, junior doctors who attended their weekly teaching sessions were given a questionnaire exploring their confidence on: finding an incident form, completing an incident form, and knowing what to report as an incident. Within the questionnaire was a free text box for them to comment on their understanding of what should be reported as an incident, as well as asking them about any incidents they had reported whilst working in the Trust. At this time, they were unaware that the hospital was planning an "Incident Reporting Week."

Following this assessment, the doctors were given a tutorial on Datix, the incident reporting tool that the hospital uses (and is widely used throughout the world) by one of the Datix team. The tutorial consisted of teaching on how to actually report an incident and how incident reporting is used throughout the hospital.

Through discussion with junior doctors about where they felt the highest risks occurred, and consultation with senior clinicians involved in quality and safety, a range of topics were chosen for the junior doctors to focus their incident reporting on. These were: VTE assessment and prescription errors, any issues relating to warfarin prescribing, and any issues with communication (with particular focus on late alerting of patient deterioration). All doctors were encouraged to report an incident during the following week, with email reminders sent before and during this period.

Following the incident reporting period, junior doctors who attended their weekly teaching sessions were given the same questionnaire on their knowledge of the incident reporting system to complete. The incidents that were reported during the reporting week were also discussed with them, along with the plans developed to address these. Data were stored and analysed on Microsoft Excel $\mathrm{XP}$.

\section{Results}

In the seven months from August 2013 to February 2014, 15 incidents were reported by junior doctors. During 'Incident Reporting Week', 17 incidents were logged by junior doctors. In the five months following the education and increased awareness of incident reporting week, 76 incidents have been logged by junior doctors (figure 1).

\section{Junior doctor reporting and confidence}

Thirty three juniors completed the questionnaires prior to 'Incident Reporting Week' ( $F 1 n=23, F 2 n=10)$. Of these only seven (21.2\%) had ever completed an incident form in this Trust. Of these, five (71.4\%) doctors were F1s and two (28.6\%) were F2s. Of these, none had received feedback regarding the incident they reported.

The questionnaire asked if the juniors had ever been shown how to complete an incident form. Prior to the intervention only two (6.06\%) doctors stated yes, however after the incident reporting week 16 $(61.5 \%)$ of doctors stated yes (figure 2$)$. When all junior doctors were asked whether they were aware of anyone receiving feedback regarding a reported incident, the response was usually negative. Only two (6.1\%) stated "yes" pre-intervention and three (11.5\%) post-intervention (figure 3 ).

The next section of the questionnaire covered confidence surrounding reporting incidents. Figure 4 demonstrates the change in confidence regarding finding the incident form. Pre-intervention, over half $(66.6 \%)$ of junior doctors were not confident or unsure about finding an incident form. Following the intervention only 
$19.2 \%$ of doctors asked were unsure about finding an incident form.

Figure 5 demonstrates the change in confidence regarding completing an incident form. Pre-intervention, over half (57.6\%) of junior doctors were unsure about finding an incident form, compared to only $15.4 \%$ post-intervention.

Figure 6 demonstrates the change in confidence regarding knowing what to report as an incident. Pre-intervention only $6.06 \%$ of junior doctors were fairly confidence about what should be reported as an incident, with no one stating they were very confident. Postintervention, over half $(53.8 \%)$ stated they either fairly or very confident about what should be reported as an incident.

Direct feedback from junior doctors who were part of this project included statements such as: "I feel more confident when reporting an incident, knowing that the hospital is not looking for blame but how to improve a current system" and "I feel like an issue that I raise through the reporting system will be listened to and acted upon appropriately".

The number of incidents reported around medication safety $(n=9)$ and particularly focused on warfarin prescribing $(n=7)$ provided evidence, rather than anecdote, that our systems around warfarin prescribing could be improved. An improvement project involving the senior pharmacist and delivery of point of care testing has been established through the use of this information.

See supplementary file: ds4113.docx - "Figures Post Peer Review"

\section{Lessons and limitations}

Overall, this project demonstrated that having an incident reporting programme and action week increased not only the number of reports, but also positively impacted the doctors' confidence about recognising an incident and completing and finding the incident form.

We targeted the junior doctors by attending their weekly teaching sessions. Therefore we did not capture all the doctors as some would have been on annual leave or unable to attend teaching due to clinical pressures. In order to capture more junior doctors we would need to attend more than just one session. Additionally, we could introduce this teaching to new doctors at their Trust induction.

This project has shown that increased teaching and awareness of incident reporting targeted at one staff group within the Trust is manageable, and has a positive outcome on the hospital and the systems within it. However, we clearly have work to do to improve our feedback on learning from incidents and that is the subject of further work in this area. Strategies to maintain sustainability of reporting such as regular focussed incident reporting weeks should be considered.[11] Additionally further development to the programme to identify other potential barriers to reporting needs to be investigated, including the creation and preservation of a blamefree culture for reporting especially among young doctors, and the influence that senior role models speaking about their own failures would have on junior doctors.[4,13]
This training should not be concentrated purely on junior doctors but should be given to all new doctors, of any grade, to the Trust. Such training could be attached to the mandatory e-learning that is already in place.

\section{Conclusion}

The principal aim of this project was to increase junior doctor's knowledge and understanding of incident reporting. Results revealed that focussed teaching and education on incident reporting increased the occurrence of reports completed by junior doctors. Prior to "Incident Reporting Week", on average only two reports were submitted a month compared with an average of 16 per month following the education and awareness of incident reporting week.

This project has demonstrated that a simple, focussed education/teaching session as an educational tool to all new junior doctors to the Trust improves incident reporting; incident reporting rates have remained elevated suggesting that junior doctors have an increased awareness of the value of incident reporting.

\section{References}

1. Institute of Medicine. To Err Is Human: Building a Safer Health System. In: Kohn L, Corrigan J, Donaldson M, eds. Washington, DC: National Academies Press, 1999:86e108.

2. Baruch N. Adverse incidents and patient safety- improving the learning experience of junior doctors. Clin Med 2014. 14:42-3.

3. Schectman JM , Plews-Ogan ML, Physician perception of hospital safety and barriers to incident reporting. Jt Comm J Qual Patient Saf 2006 Jun. 32(6):337-43.

4. Evans SM, Berry JG, Smith BJ, Esterman A, Selim P, O'Shaughnessy J, DeWit M. Attitudes and barriers to incident reporting: a collaborative hospital study. Qual Safe Health Care 2006. 15:39-43.

5. Lawton R, Parker D. Barriers to incident reporting in a healthcare system. Qual Saf Healthcare 2002. 11:15-8.

6. Larizgoitia I, Bouesseau MC, Kelley E. WHO Efforts to Promote Reporting of Adverse Events and Global Learning. J Public Health Res 2013 Dec 1. 2(3):e29.

7. Hudson P. Applying the lessons of high risk industries to health care. BMJ Qual Saf Health Care 2003. 12:i7-i12 doi:10.1136/qhc.12.suppl_1.i7.

8. Hutchinson A, Young TA, Cooper KL, McIntosh A, Karnon JD, Scobie S, Thomson RG. Trends in healthcare incident reporting and relationship to safety and quality data in acute hospitals: results from the National Reporting and Learning System. Qual Saf Health Care 2009. 18;5-10.

9. Leape L. Reporting of adverse incidents. NEJM 2002. 347:1633-8.

10. Vincent C, Stanhope N, Crowley-Murphy M. Reasons for not reporting adverse incidents: an empirical study. J Eval Clin Pract 1999. 5:13-21.

11. Pham JC, Girard T, Pronovost PJ. What to do with healthcare incident reporting systems. J Pub Health Res 2013;2:e27. 


\section{BMJ Quality Improvement Reports}

12. Higginson J, Walters R, Fulop N. Mortality and morbidity meetings: an untapped resource for improving the governance of patient safety? BMJ Qual Saf 2012. doi:10.1136/bmjqs-2011-00060.

13. Hewitt T, Chreim S, Forster A. Sociocultural Factors Influencing Incident Reporting Among Physicians and Nurses: Understanding Frames Underlying Self- and PeerReporting Practices. J Patient Saf 2014. Epub ahead of print. doi:10.1097/PTS.0000000000000130.

14. World Alliance for Patient Safety. WHO draft guidelines for adverse event reporting and learning systems: from information to action. Geneva: World Health Organization; 2005.

15. Pham JC, Gianci S, Battles J, et al. Establishing a global learning community for incident-reporting systems. Qual Saf Health Care 2010. 19:446-51. doi:10.1136/qshc.2009.037739.

16. Keogh B. Review into the quality of care and treatment provided by 14 hospital trusts in England: overview report. http://www.nhs.uk/NHSEngland/bruce-keoghreview/Documents/outcomes/keogh-review-final-report.pdf (accessed 11/8/2014).

\section{Declaration of interests}

Nothing to declare.

\section{Acknowledgements}

Debbie Gale, risk application manager. 